\title{
Impact of gender, C-reactive protein and body mass index on erythropoietin resistance index in maintenance hemodialysis patients
}

\author{
Asim Osman $^{1 \oplus}$, Nada Awad Alkareem² $^{2}$ Baha eldin Elawad ${ }^{3}$, Ogail Dawod $^{4}$, Mohammed Elshiekh $^{5 *}$
}

\begin{abstract}
Introduction: Anemia is caused by a variety of mechanisms in chronic kidney disease (CKD), including erythropoietin (EPO) deficiency, resistance to erythropoiesis-stimulating agents (ESAs), impaired iron metabolism and its clinical management remains challenging.

Objectives: The aim of the current study was to evaluate the impact of CRP, BMI, gender and duration of hemodialysis.

Patients and Methods: A total of 94 maintenance HD patients participated in this study. Laboratory investigation included CBC, renal function test and qualitatively C-reactive protein was performed. Erythropoietin resistance index (ERI) was calculated as weekly EPO dose/ body weight in $\mathrm{kg} / \mathrm{hemoglobin}$ level.

Results: Female gender had significantly higher ERI $(11.36 \pm 1.52)$ compared to male HD patients $(10.68 \pm 1.56)(P>0.05)$. Patients with low BMI had significant higher ERI (12.08 \pm 1.09$)$ compared to HD patients with overweight (10.62 \pm 0.79$)$ and obese $(9.62 \pm 1.68)(P>$ $0.05)$. The highest ERI were found in the positive CPR group $(P>0.05)$ compared to negative CRP group. There is no significant difference between duration of hemodialysis.

Conclusion: Our data exposed that female gender; low BMI and inflammation (positive CRP) contributed to EPO hyporesponsiveness. In addition, there is no significant difference between lengths on hemodialysis.

Keywords: Hemodialysis, Erythropoietin, Hyporesponsiveness, C-reactive protein, Anemia

Citation: Osman A, Alkareem NA, Elawad B, Dawod O, Elshiekh M. Impact of gender, C-reactive protein and body mass index on erythropoietin resistance index in maintenance hemodialysis patients. J Renal Endocrinol. 2021;7:e04. doi: 10.34172/jre.2021.04. Copyright $\odot 2021$ The Author(s); Published by Nickan Research Institute. This is an open-access article distributed under the terms of the Creative Commons Attribution License (http://creativecommons.org/licenses/by/4.0), which permits unrestricted use, distribution, and reproduction in any medium, provided the original work is properly cited.
\end{abstract}

\section{Introduction}

Erythropoietin resistance index (ERI) calculation has been used to find out the degree of responsiveness to erythropoietin (EPO). Previous studies documented that there are many factors have been shown to independently increase ERI include male sex, shorter period on dialysis, low body mass index (BMI), low serum albumin level, low iron saturation, higher parathyroid hormone (PTH) levels, and the use of angiotensin-converting enzyme inhibitors/ angiotensin receptor blockers (1-3).

The sensitivity of the ERI enables the prompt detection of primary variations (4). The most ordinarily cause of ERI change is inflammation and malnutrition, ignoring iron deficiency. ERI has been considered a good marker for predicting mortality in dialysis patients. The BMI is a factor in the modification of body response to EPO (3). However, whether or not this factor is solely or partially responsible for ERI change involves to be investigated. Hence, hypo-responsiveness to EPO has also been shown to be related to increased hospitalization and mortality.
The highest ERI tertile was related to higher all-cause mortality (hazard ratio 4.2) (5). Interestingly, this association between all-cause mortality and ERI is seen in HD patients but not in PD patients (6).

Inflammation is prevalent in $\mathrm{HD}$ patients. As an inflammatory marker, C-reactive protein (CRP) is an important sensitive acute phase reactant (7). Previous researches manifested that elevated level of serum CRP is a strong predictor of cardiovascular mortality both in healthy subjects and HD patients $(8,9)$.

In addition to being a prognostic indicator, elevated level of CRP may also affect responsiveness to EPO therapy in HD patients. Previous studies in small samples have shown a strength correlation between elevated levels of inflammation and hypo-responsiveness to ESAs (10, 11 ), as measured by total ESA dose requirements.

\section{Objectives}

Regarding the great inter-patient variability of administered EPO doses needed to maintain patients 
Implication for health policy/practice/research/ medical education

ESAs are the main treatment used to manage anemia in HD patients. The response ESAs in patients with CKD is variable and can be modified in several circumstances. The ERI is considered to be a helpful parameter in assessing the body's response to ESA. However, an association between higher ESA doses and mortality has been observed in many interventional studies. In recent years, there has been growing interest in defining the effective factors and optimal ESA doses that will help patients reach the hemoglobin target without increasing their mortality.

at particular hemoglobin levels $(12,13)$, evaluating the influence of inflammation on hemoglobin response to ESA dose is important for guiding anemia management. So far few studies have had adequate sample size and collection of factors that may influence ESA dosing to evaluate the independent role of inflammation, BMI on ERI. Therefore, the aim of the current study was to evaluate the impact of CRP, BMI, gender and duration of hemodialysis that may contribute to reduce responsiveness to ESA in patients on regular hemodialysis.

\section{Patients and Methods}

\section{Study design}

This was a descriptive retrospective cross-sectional study, conducted among maintenance hemodialysis patients in North State, Sudan in the following dialysis centers Kariam, Merowe and Nori centers. 94 Sudanese hemodialysis patients, aged 25-60 years diagnosed as an ESRD were included randomly. The current study was approved by the ethics committee of Ministry of Health, Northern State, Sudan. Written informed consent from participants was obtained prior to study.

Demographic data, time of dialysis per week, length (duration) on dialysis, administration of EPO and dose of EPO (Unit/week) were recorded. Height and weight were measured and BMI was calculated as weight $/$ height $^{2}$ $\left(\mathrm{kg} / \mathrm{m}^{2}\right)$.

In all patients, the HD procedure was carried out by using dialyzers containing high flux membranes such as polysulfone, polyester-polymer alloy, or cellulose triacetate at a blood flow rate of $200-250 \mathrm{~mL} / \mathrm{min}$ and a dialysate flow rate of $500 \mathrm{~mL} / \mathrm{min}$. The surface area of the dialyzer membrane was chosen according to patient body weight. The glucose concentration of the dialysate was $100 \mathrm{mg} / \mathrm{dL}$. Heparin was administered at 2600-5000 $\mathrm{U}$ per 4-h HD session for anticoagulation. The volume of ultrafiltration was maintained on the basis of clinical dry weight during each session.

\section{Blood tests}

Five milliliters of blood was drawn from patients during the first 15 minutes of hemodialysis For CBC blood
Samples were processed using EDAN CBC fully auto hematology analyzer machine. Total and differential white blood cells (WBC) counts and percentages were determined. The absolute counts for the neutrophils, eosinophils, basophils, lymphocytes, and monocytes were computed from the WBCs total counts and the differential counts (\%) of each type of white blood cells. In addition, red blood cells (RBCs) count, hematocrit percentage (HCT \%), hemoglobin content, and platelets counts were determined in each blood sample.

ERI was defined as the average weekly ESA dose divided by clinical dry weight and average blood hemoglobin (weekly ESA dose (units)/dry weight (kg)/hemoglobin (g/ $\mathrm{dL})$ ), as described previously (14).

For determination of Renal Function Tests, measurement of serum and urine creatinine was done using Newman and Price method using BioSystems reagent kits. Measurement of serum urea was done by colorimetric method using end-point determination Urease-Berthelot Reaction, using BioSystems reagent kits. Measurement of serum sodium, potassium, calcium and phosphate was done using the flame photometer. For estimation of serum, CRP blood samples were collected in plain container. CRP levels were detected (qualitatively) using latex agglutination method (Spinreact kit, Spain).

\section{Ethical issues}

Before starting the study, the patients were informed about the aims, the expected benefits to them and/or others, the risks and inconveniences involved, and their right to refuse to participate or to withdraw from the study at any time without sanction. Their written consent was obtained. The study was carried out in accordance with the Declaration of Helsinki and its subsequent modifications, and was approved by the Ethics Committees of Ministry of Health, Northern State, Sudan (approval number \#451). This study was extracted from MS.c thesis of Nada Awad Alkareem at Merowe University of Technology.

\section{Statistical analysis}

Data was analyzed by using SPSS software version 24 (SPSS Inc, IL, USA). Data were presented as mean \pm standard deviation for quantitative variables and summarized as frequencies and percentages for categorical variables. Student $t$ test was used to compare between two groups. Data were analyzed using one-way ANOVA followed by post hoc Tukey's test. $P$ value of less than 0.05 was considered significant.

\section{Results}

A total $94 \mathrm{CKD}$ patients maintenance on $\mathrm{HD}$ were included in this study. The demographics and clinical data of maintenance HD patients participated as subjects were shown in Table 1.

Our results demonstrated that female HD patients had significantly higher ERI $(11.36 \pm 1.52)$ compared to male 
Table 1. Demographics and clinical data study participants

\begin{tabular}{|c|c|c|c|}
\hline Variables & & Mean or (n) Percent & SD \\
\hline \multirow{2}{*}{ Gender } & Male & (57) $60.6 \%$ & \\
\hline & Female & (37) $39.4 \%$ & \\
\hline \multirow{3}{*}{ BMI } & Normal weight & (75) $79.8 \%$ & \\
\hline & Over weight & (12) $12.8 \%$ & \\
\hline & Obese & (7) $7.4 \%$ & \\
\hline \multirow{2}{*}{ CRP } & Positive & (45) $47.9 \%$ & \\
\hline & Negative & (49) $52.1 \%$ & \\
\hline \multirow{2}{*}{ EPO use } & Use & (88) $93.6 \%$ & \\
\hline & Did not use & (6) $6.4 \%$ & \\
\hline \multirow{2}{*}{$\begin{array}{l}\text { Duration of } \\
\text { dialysis }\end{array}$} & $<36$ months & (55) $58.5 \%$ & \\
\hline & $>36$ months & (39) $41.5 \%$ & \\
\hline Height & & 162.67 & 16.31 \\
\hline Weight & & 59.93 & 15.60 \\
\hline $\mathrm{Hb}(\mathrm{g} / \mathrm{dL})$ & & 9.94 & 2.14 \\
\hline RBCs (x10\%/uL) & & 4.0077 & 3.48 \\
\hline PCV (\%) & & 33.93 & 9.34 \\
\hline $\mathrm{MCV}\left(\mathrm{um}^{3}\right)$ & & 91.18 & 6.52 \\
\hline $\mathrm{MCH}(p g)$ & & 29.37 & 9.62 \\
\hline MCHC (g/dL) & & 31.80 & 7.34 \\
\hline ERI & & 10.95 & 1.56 \\
\hline WBCs $\left(\times 10^{3} / u L\right)$ & & 6.013 & 2.325 \\
\hline PLT (x103/uL) & & 223.37 & 72.67 \\
\hline Creatinine (mg/dL) & & 8.05 & 2.81 \\
\hline Urea (mg/dL) & & 116.37 & 4.19 \\
\hline $\mathrm{PO}_{4}(\mathrm{mg} / \mathrm{dL})$ & & 5.19 & 1.69 \\
\hline $\mathrm{Ca}^{2+}(\mathrm{mg} / \mathrm{dL})$ & & 8.87 & 1.27 \\
\hline $\mathrm{K}^{+}(\mathrm{mmol} / \mathrm{L})$ & & 5.24 & 1.02 \\
\hline $\mathrm{Na}^{+}(\mathrm{mmol} / \mathrm{L})$ & & 134.26 & 6.26 \\
\hline
\end{tabular}

Data presented as (frequency) percent or mean \pm S.D. BMI; body mass index. CRP; C-reactive protein. EPO; erythropoietin. $\mathrm{Hb}$; hemoglobin. RBCs; red blood cells. PCV; package cell volume. MCV; mean corpuscle volume. $\mathrm{MCH}$; mean hemoglobin concentration. MCHC; mean corpuscle of hemoglobin concentration. ERI; erythropoietin resistance index. WBCs; white blood cells. PLT; platelet.

HD patients $(10.68 \pm 1.56)(P>0.05$; Figure $1 \mathrm{~A})$.

HD patients with normal weight had significant higher ERI $(12.08 \pm 1.09)$ compared to HD patients with overweight $(10.62 \pm 0.79)$ and obese $(9.62 \pm 1.68)(P>$ $0.05)$. In addition, $H D$ patients with overweight had significant higher ERI compared to obese HD patients ( $P$ $>0.05$ ) (Figure 1B).

In our results, we divided $\mathrm{HD}$ patients into two groups on the basis of qualitative CPR and found significant difference in ERI. The highest ERI were found in the positive CPR group ( $P>0.05$; Figure $2 \mathrm{~A})$.

Patients with length on dialysis less than 36 months had lower ERI (10.87 \pm 1.25$)$ compared to patients with length on dialysis more than 36 months but there is no significant difference between two lengths (Figure 2B).
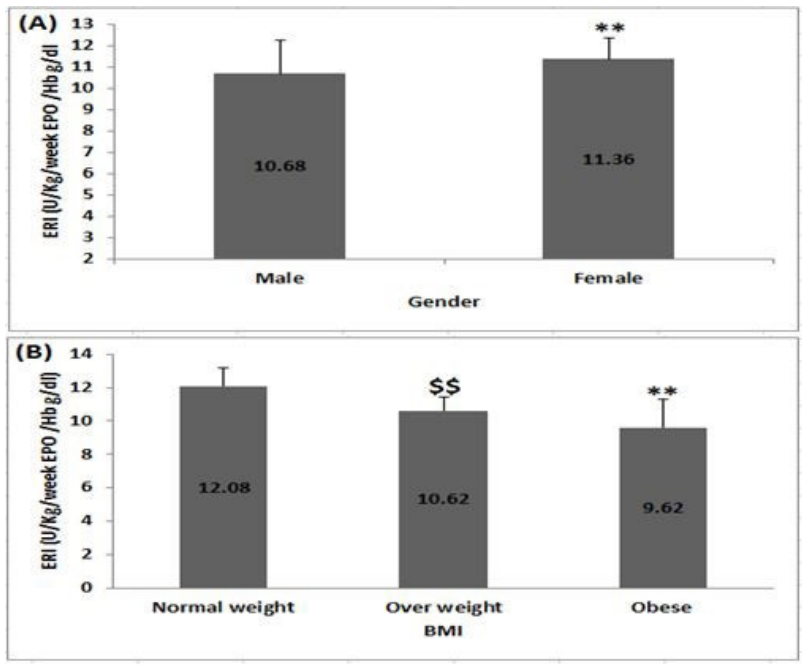

Figure 1. Impact of gender (A) and BMI (B) on ERI. Data represent as mean \pm S.D. ERI: Erythropoietin resistance index; ${ }^{* *} P>0.05$ vs Male group; ${ }^{* *} P>$ 0.05 vs normal weight and overweight; ${ }^{\oplus} P>0.05$ vs normal weight.

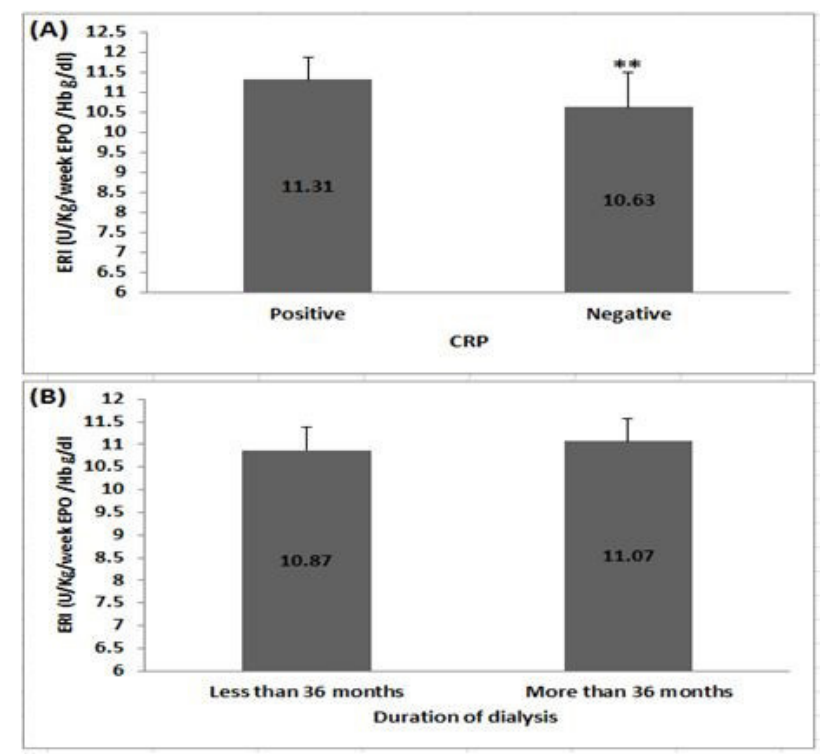

Figure 2. Impact of C-reactive protein (A) and duration of hemodialysis (B) on ERI. Data represent as mean \pm S.D. ${ }^{* *} P>0.05$ vs positive CRP group. ERI: erythropoietin resistance index; CRP: C-reactive protein.

Our data showed that there is no significant difference in hemoglobin levels (Figure 3A) and mean corpuscle hemoglobin concentration (Figure $3 \mathrm{~B}$ ) between positive and negative CRP groups. In addition, we demonstrated that there is no significant difference in hemoglobin levels between BMI categories (Figure 3C).

\section{Discussion}

Anemia is caused by a variety of mechanisms in CKD, including erythropoietin deficiency, resistance to ESAs, impaired iron metabolism and its clinical management remains challenging. The aim of the current study was to evaluate the impact of CRP, BMI, gender and duration of 


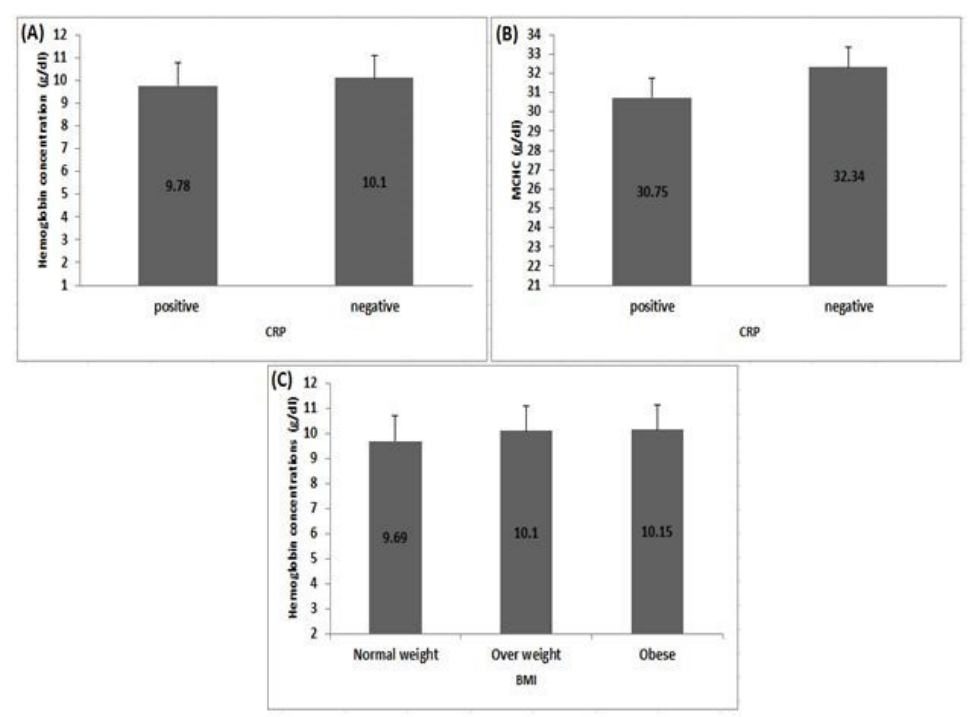

Figure 3. Impact of CRP on hemoglobin (A) levels and MCHC (B). Impact of BMI (C) on hemoglobin levels. Data represent as mean \pm S.D. CRP; C-reactive protein; MCHC: mean corpuscle of hemoglobin concentration; BMI: body mass index.

hemodialysis. The finding of our current study the mean of ERI was $10.95 \pm 1.56 \mathrm{U} / \mathrm{kg} /$ week $\mathrm{EPO} / \mathrm{Hb} \mathrm{g} / \mathrm{dL}$. In addition, our data showed that male sex had higher ERI than female; patients with normal weight category of BMI had significant higher ERI than other BMI categories and patients with positive CRP had significant elevated ERI than patients with negative CRP. In our current study, we found that patients with duration of hemodialysis more than 36 months had higher ERI than patients with duration of hemodialysis less than 36 months but there is not reach significant value.

EPO has been applied in the clinic for many decades and has greatly improved anemia, EPO hyporesponsiveness is still observed in some HD patients. Previous studies have documented a relation between EPO hyporesponsiveness and poor clinical outcomes, such as anemia, heart failure, and increased cardiovascular and all-cause mortality $(15,16)$. The ERI is a sensitive evaluation index of EPO responsiveness and can predict composite events (CVD, infection, hospitalization, or death) (16) and all-cause mortality in HD patients $(17,18)$. Our current study showed that ERI values were from 8.02 to $13.72 \mathrm{U} / \mathrm{kg} /$ week $\mathrm{EPO} / \mathrm{Hb} \mathrm{g} / \mathrm{dL}$, and the mean ERI value was $(10.95 \pm$ 1.56) $\mathrm{U} / \mathrm{kg} /$ week EPO/Hb g/dL. These data are consistent with data reported by other studies $(3,19)$.

Recent studies have established considerable variability in ESA dose requirements among hemodialysis patients $(12,20)$. Inflammatory conditions, already established as an important factor limiting the response to ESA treatment in hemodialysis patients, are the probable cause of the positive association that exists between ferritin and the ERI $(21,22)$. Gunnell et al demonstrated that elevated level of CRP is associated with the highest EPO dose needs for any given hemoglobin concentration (23). A study done by Locatelli and colleagues showed that a strong association between CRP levels and both absolute EPO dose and the ERI, even after adjustment for many potential confounding factors (24). Our current study demonstrated that HD patients with positive CRP had elevated ERI than patients with negative CRP. In addition, we demonstrated that negative CRP patients had greater hemoglobin concentration and MCHC than positive CRP patients, but there was not reach significant value.

Since the proposed of EPO in the late 1980s, it has been possible for HD patients to achieve desirable Hemoglobin concentrations. As a result, the mean Hemoglobin level in HD patients has improved significantly over the past two decades (25). EPO achieving dosing can be challenging in HD patients due to variations in patient response. In our current study mean of ERI in normal weight patients was significantly higher than overweight and obese patients. In addition, the mean of ERI in overweight patients was significantly higher than obese patients. The results of our study similar to previous study reported that the median ESA dose and ERI value were significantly higher in non-obese than obese patients (26). This rising in ERI in normal weight patients did not seem to result in worsens anemia control. We documented that there is no significant difference in hemoglobin levels among patients in the different BMI categories. A variety of factors have been described to impact the responsiveness to EPO in HD patients, including secondary hyperparathyroidism, iron deficiency, systemic inflammation, dialysis adequacy and malnutrition $(1,15,27)$.

The higher ERI in some patients is a condition that has been studied. A study on low response to EPO established that female receive higher EPO doses to attain the hematocrit target. It also found a higher proportion of female receiving EPO doses, but no association between iron levels or high levels of PTHs and the use of EPO 
doses (28). Other publications have found that women require higher doses of $\mathrm{EPO}$, or even that low response is associated with iron deficiency, poor nutritional state, high-turnover bone disease, and tunneled-catheter vascular access $(3,29)$. Our current study demonstrated that the ERI was greater in female HD patients than male. Our findings concur with other publications observing a relation between gender and EPO requirements. A study assessing the association of anemia and survival found that the masculine gender was one of the predictors of higher levels of Hemoglobin, with an inverse relationship between hemoglobin levels and EPO doses.

Physiologically, women have lower hemoglobin levels than men, which are attributed to the effect of androgens and estrogens on erythropoiesis. This occurs mainly because of the vasodilator effect that estrogens have on the kidney microvasculature, which causes higher oxygen liberation per red blood cells mass unit at a juxtaglomerular apparatus level (30). According to the World Health Organization, there are gender-associated differences in the hemoglobin levels that are considered normal, as well as in the hemoglobin thresholds used to define anemia, which is also applicable to patients with CKD (31). Despite the differences between normal levels of hemoglobin and the anemia cut-off, the current guidelines of anemia management in patients with CKD establish a hemoglobin target with no gender distinction. A study conducted on a population that was not undergoing dialysis or receiving EPO reported that there is a higher absolute hemoglobin level in men than in women in different stages of CKD. They proposed considering a relative gender-specific hemoglobin level defined as the percentage of measured hemoglobin value in relation to the normal hemoglobin inferior limit for each gender and found that relative hemoglobin is higher in women than in men. They considered that this finding was worth taking into account in future recommendations and suggested the establishment of a lower hemoglobin target in women (32). Our findings regarding the fact that women required higher doses of EPO to achieve the hemoglobin target could be the result of considering women values of hemoglobin levels as similar to men and might not indicate a difference in responsiveness to EPO.

\section{Conclusion}

Our data exposed that female gender, low BMI and inflammation (positive CRP) contributed to EPO hyporesponsiveness. In addition, there is no significance different between lengths on hemodialysis

\section{Limitations of the study}

The limitations of our study include the fact that its crosssectional design means that it can only estimate the effect of the explored variables, but cannot investigate the direction of the link between the cause (gender, BMI and CRP) and EPO resistance. Secondly, as a single-center (State) cross- sectional study, our data may not be representative of MHD patients in other states or other countries. Thirdly, given that we detected CRP qualitatively.

\section{Acknowledgments}

The authors thank patients for their participation. We would like to thank staff and administration of dialysis centers Kariam, Merowe and Nori for their cooperation.

\section{Authors' contribution}

NAA and AO were the principal investigators of the study. ME, $\mathrm{AO}, \mathrm{BE}$ and NAA were participated in preparing the concept and design of study. ME, BE and OD analyzed data and revised the final manuscript. All authors participated in preparing the final manuscript, revised the manuscript and critically evaluated the intellectual concepts. All authors have read and approved the content of the manuscript and confirmed the accuracy or integrity of any part of the work.

Conflicts of interest

There are no conflicts of interest.

Ethical considerations

Ethical issues (including plagiarism, data fabrication, double publication) have been completely observed by the authors.

\section{Funding/Support}

This study was carried out without any financial support from any company.

\section{References}

1. MallickMallick S, Rafiroiu A, Kanthety R, Iqbal S, Malik $R$, Rahman M. Factors predicting erythropoietin resistance among maintenance hemodialysis patients. Blood Purif. 2012; 33:238-44. doi: 10.1159/000335256.

2. Schneider A, Schneider MP, Scharnagl H, Jardine AG, Wanner C, Drechsler C. Predicting erythropoietin resistance in hemodialysis patients with type 2 diabetes. BMC Nephrol. 2013;14:67. doi: 10.1186/1471-2369-14-67.

3. López-Gómez JM, Portolés JM, Aljama P. Factors that condition the response to erythropoietin in patients on hemodialysis and their relation to mortality. Kidney Int Suppl. 2008;111:S75-81. doi: 10.1038/ki.2008.523

4. Kaysen GA, Muller HG, Ding J, Chertow GM. Challenging the validity of the EPO index. Am J Kidney Dis. 2006;47:166. e1-166.e13. doi: 10.1053/j.ajkd.2005.09.013.

5. Eriguchi R, Taniguchi M, Ninomiya T, Hirakata H, Fujimi S, Tsuruya K. Hyporesponsiveness to erythropoiesis-stimulating agent as a prognostic factor in Japanese hemodialysis patients: The Q-Cohort study. J Nephrol 2015;28:217-25. doi: 10.1007/ s40620-014-0121-9

6. Bae MN, Kim SH, Kim YO, Jin DC, Song HC, Choi EJ, et al. Association of erythropoietin-stimulating agent responsiveness with mortality in hemodialysis and peritoneal dialysis patients. PLoS One. 2015;10:e0143348. doi: 10.1371/journal. pone. 0143348.

7. Owen WF, Lowrie EG. C-reactive protein as an outcome predictor for maintenance hemodialysis patients. Kidney Int. 1998;54(2):627-636. doi: 10.1046/j.1523-1755.1998.00032.x

8. Ridker PM, Cushman M, Stampfer MJ, Tracy RP, Hennekens CH. Inflammation, aspirin, and the risk of cardiovascular disease in apparently healthy men. N Engl J Med. 1997;336(14):973979. doi: 10.1016/S1053-0770(97)90146-4

9. Zimmermann J, Herrlinger S, Pruy A, Metzger T, Wanner C. Inflammation enhances cardiovascular risk and mortality in 
hemodialysis patients. Kidney Int. 1999;55(2):648-58. doi: 10.1046/j.1523-1755.1999.00273.x.

10. Rossert J, Gassmann-Mayer C, Frei D, McClellan W. Prevalence and predictors of epoetin hyporesponsiveness in chronic kidney disease patients. Nephrol Dial Transplant. 2007;22: 794-800. doi: 10.1093/ndt/gfl716

11. Singh AK, Coyne DW, Shapiro W, Rizkala AR. Predictors of the response to treatment in anemic hemodialysis patients with high serum ferritin and low transferrin saturation. Kidney Int. 2007;71:1163-71. doi: 10.1038/sj.ki.5002223

12. Zhang Y, Thamer M, Stefanik K, Kaufman J, Cotter DJ. Epoetin requirements predict mortality in hemodialysis patients. Am J Kidney Dis. 2004;44:866-76. doi: 10.1053/j. ajkd.2004.08.002.

13. Bradbury BD, Critchlow CW, Weir MR, Stewart R, Krishnan M, Hakim RH. Impact of elevated C-reactive protein levels on erythropoiesis- stimulating agent (ESA) dose and responsiveness in hemodialysis patients. Nephrol Dial Transplant. 2009;24:919-25. doi: 10.1093/ndt/gfn543.

14. Higuchi T, Abe M, Yamazaki T, Mizuno M, Okawa E, Ando $\mathrm{H}$, et al. Effects of levocarnitine on brachial-ankle pulse wave velocity in hemodialysis patients: a randomized controlled trial. Nutrients 2014;6:5992-6004. doi: 10.3390/nu6125992

15. Szczech LA, Barnhart HX, Inrig JK, Reddan DN, Sapp S, Califf RM, et al. Secondary analysis of the CHOIR trial epoetinalpha dose and achieved hemoglobin outcomes. Kidney Int. 2008;74(6):791-8. doi: 10.1038/ki.2008.295.

16. Kilpatrick RD, Critchlow CW, Fishbane S, Besarab A, StehmanBreen C, Krishnan M, et al. Greater epoetin alfa responsiveness is associated with improved survival in hemodialysis patients. Clin J Am Soc Nephrol. 2008;3:1077-83. doi: 10.2215/ CJN.04601007.

17. Kuragano T, Kitamura K, Matsumura O, Matsuda A, Hara T, Kiyomoto $\mathrm{H}$, et al. ESA Hyporesponsiveness Is Associated with Adverse Events in Maintenance Hemodialysis (MHD) Patients, But Not with Iron Storage. PLoS One. 2016;11:e0147328. doi: 10.1371/journal.pone.0147328

18. Okazaki M, Komatsu M, Kawaguchi H, Tsuchiya K, Nitta K. Erythropoietin resistance index and the all-cause mortality of chronic hemodialysis patients. Blood Purif. 2014;37:106-12. doi: 10.1159/000358215

19. Chang JH, Jung JY, Lee HH, Chung W, Joo KW, Kim S. Serum resistin as a novel marker of erythropoietin resistance in nondiabetic patients on hemodialysis. Tohoku J Exp Med. 2011;224:281-5. doi: 10.1620/tjem.224.281

20. Bradbury BD, Critchlow CW, Rothman KJ, Heagerty P, Keen M, Acquavella JF, et al. Exploring relative mortality and epoetin alfa among hemodialysis patients. Am J Kidney Dis 2008 Jan
1; 51: 62-70. doi: 10.1053/j.ajkd.2007.09.015

21. Romão Jr JE, Haiashi AR, Elias RM, Luders C, Ferraboli R, Castro MCM, et al. Positive Acute-Phase Inflammatory Markers in Different Stages of Chronic Kidney Disease. Am J Nephrol 2006;26:59-66. doi: 10.1159/000091806

22. Abensur H. Iron deficiency in chronic kidney disease. Rev. Bras. Hematol. Hemoter. 2010 Jun; 32 (Suppl 2): 95-98. doi: 10.1590/S1516-84842010005000047

23. Gunnell J, Yeun JY, Depner TA, Kaysen GA. Acute-phase response predicts erythropoietin resistance in hemodialysis and peritoneal dialysis patients. Am J Kidney Dis 1999;33: 63-72. doi: 10.1016/S0272-6386(99)70259-3

24. Locatelli F, Andrulli S, Memoli B, Maffei M, Vecchio LD, Aterini $S$, et al. Nutritional-inflammation status and resistance to erythropoietin therapy in haemodialysis patients. Nephrol Dial Transplant 2006;21:991-8. doi: 10.1093/ndt/gfk011.

25. Freburger JK, Ng LJ, Bradbury BD, Kshirsagar AV, Brookhart MA. Changing patterns of anemia management in US hemodialysis patients. Am J Med. 2012;125:906-914.e9, 2012. doi: 10.1016/j.amjmed.2012.03.011.

26. El-Kannishy GM, Megahed AF, Tawfik MM, El-Said G, Zakaria RT, Mohamed NA, et al. Obesity may be erythropoietin dose-saving in hemodialysis patients. Kidney Res Clin Pract. 2018;37(2):148-56. doi: 10.23876/j.krcp.2018.37.2.148.

27. Di lorio BR, Stellato D, De Santo NG, Cirillo M. Campanian Dialysis Registry Research Group. Association of gender and age with erythropoietin resistance in hemodialysis patients: role of menstrual status. Blood Purif. 2004;22:423-7. doi: $10.1159 / 000080234$.

28. Eschbach JW, Varma A, Stivelman JC. Is it time for a paradigm shift? Is erythropoietin deficiency still the main cause of renal anemia? Nephrol Dial Transplant 2002;17 Suppl 5:2-7. doi: 10.1093/ndt/17.suppl_5.2.

29. Kalantar-Zadeh K, Lee GH, Miller JE, Streja E, Jing J, Robertson JA, et al. Predictors of hyporesponsiveness to erythropoiesis-stimulating agents in hemodialysis patients. Am J Kidney Dis 2009;53:823-34. doi: 10.1053/j.ajkd.2008.12.040

30. Murphy WG. The sex difference in haemoglobin levels in adults - Mechanisms, causes, and consequences. Blood Rev. 2014;28:41-7. doi: 10.1016/j.blre.2013.12.003

31. Benoist B, McLean E, Egli I, Cogswell M. Worldwide Prevalence of Anaemia 1993-2005: WHO Global Database on Anaemia. Geneva: World Health Organization; 2008.

32. Duncan JA, Levin A. Sex, haemoglobin and kidney disease: New perspectives. Eur J Clin Invest 2005;35Suppl 3:52-7. doi: 10.1111/j.1365-2362.2005.01531.x. 